\title{
Determination of mean pressure from PIV in compressible flows using the Reynolds-averaging approach
}

\author{
Paul L. van Gent ${ }^{1}$ · Bas W. van Oudheusden ${ }^{1}$ · Ferry F. J. Schrijer ${ }^{1}$
}

Received: 12 October 2017 / Revised: 2 January 2018 / Accepted: 3 January 2018 / Published online: 8 February 2018

(c) The Author(s) 2018. This article is an open access publication

\begin{abstract}
The feasibility of computing the flow pressure on the basis of PIV velocity data has been demonstrated abundantly for lowspeed conditions. The added complications occurring for high-speed compressible flows have, however, so far proved to be largely inhibitive for the accurate experimental determination of instantaneous pressure. Obtaining mean pressure may remain a worthwhile and realistic goal to pursue. In a previous study, a Reynolds-averaging procedure was developed for this, under the moderate-Mach-number assumption that density fluctuations can be neglected. The present communication addresses the accuracy of this assumption, and the consistency of its implementation, by evaluating of the relevance of the different contributions resulting from the Reynolds-averaging. The methodology involves a theoretical order-of-magnitude analysis, complemented with a quantitative assessment based on a simulated and a real PIV experiment. The assessments show that it is sufficient to account for spatial variations in the mean velocity and the Reynolds-stresses and that temporal and spatial density variations (fluctuations and gradients) are of secondary importance and comparable order-of-magnitude. This result permits to simplify the calculation of mean pressure from PIV velocity data and to validate the approximation of neglecting temporal and spatial density variations without having access to reference pressure data.
\end{abstract}

\section{Introduction}

Knowledge of flow pressure is of fundamental importance in several fluid-dynamic areas, such as aero-acoustics, turbulence, cavitation, and fluid-structure interactions. The feasibility of obtaining pressure data from particle image velocimetry (PIV) or particle tracking velocity data has been demonstrated abundantly over the recent years, notably for low-speed conditions. The method provides an appealing approach to achieve access to the flow field pressure in a non-intrusive way. A recent overview of the operating principles and a variety of application areas has been provided in van Oudheusden (2013).

The basic working principle of the procedure is to invoke the momentum equation to calculate the local pressure gradient from flow velocity and material acceleration data, which can be provided by PIV measurements. Pressure fields are subsequently obtained through spatial integration with

Paul L. van Gent

p.1.vangent@tudelft.nl

1 Department of Aerospace Engineering, Delft University of Technology, Kluyverweg 1, 2629 HS Delft, The Netherlands appropriate boundary conditions applied. The nature of the flow configuration dictates the type of PIV data required for a successful implementation of the method: whereas planar data may be sufficient for nearly two-dimensional flows, volumetric velocity data are required for flows that are notably three-dimensional in nature (Charonko et al. 2010; Violato et al. 2011; de Kat and van Oudheusden 2012). Similarly, the determination of instantaneous pressure for unsteady flows involves the evaluation of the temporal development of the flow field, which requires high-speed (time-resolved) measurements.

It is generally accepted that the accuracy with which the material acceleration is determined is the dominant factor for the pressure reconstruction (Violato et al. 2011; de Kat and van Oudheusden 2012; van Gent et al. 2017a), setting particular demands on both spatial and temporal resolution of the velocity measurement. For application in the high-speed regime, which is the area of interest of the present communication, meeting these demands becomes increasingly challenging, for a variety of reasons. First, the environment of high-speed wind tunnel operation is characterised by increased vibration levels, limited optical access, flow seeding issues, and optical aberration effects, which adversely affect the PIV measurement accuracy. 
Furthermore, the time scales associated with high-flow speeds are beyond the current standard (high-speed) PIV capabilities, so that time-resolved data can only be obtained by adopting alternative multi-pulse procedures (Souverein and van Oudheusden 2009; Lynch and Scarano 2014; Blinde et al. 2015). Although the feasibility of using a four-pulse procedure has been validated on the basis of synthetic experimental data (van Gent et al. 2017a) and advances have been made by tracking individual tracer particles (Novara et al. 2016), achieving the accuracy required for satisfactory instantaneous pressure results is challenging (Blinde et al. 2015). Furthermore, setting up and operating a multi-pulse PIV system is significantly more complexity and laborious than using a standard double-pulse system.

Whereas the calculation of instantaneous pressure fields for unsteady flows requires time-resolved data, mean pressure fields can be derived from velocity field statistics obtained from uncorrelated velocity measurements, by means of a Reynolds-averaging approach (Gurka et al. 1999; van Oudheusden et al. 2007). Since no timeresolved measurements are required, this technique can rely on relatively simple PIV systems. Furthermore, since it is a statistical approach, it benefits from a reduction in uncertainty by combining a large ensemble of measurements. The merits of obtaining mean pressure fields from planar PIV measurements were recently demonstrated in van Gent et al. (2017b) who applied the technique to assess the impact of nozzle length and the presence of an exhaust plume on transonic and supersonic base flows with exhaust plumes. The above shows the pursuit of the mean pressure data as a worthwhile and realistic objective for high-speed flow applications.

Although the particular interest of the present study lies in pressure determination in high-speed flows which are compressible, as an introduction, first, the procedure for incompressible flow will be shortly outlined for reference (see also, e.g., Unal et al. 1997; Baur and Köngeter 1999; Gurka et al. 1999). As stated previously, the computation of pressure from the velocity field basically relies on invoking the momentum equation, which for an incompressible flow reads:

$\frac{\partial p}{\partial x_{i}}=-\rho\left(\frac{\partial u_{i}}{\partial t}+u_{j} \frac{\partial u_{i}}{\partial x_{j}}\right)+\mu \frac{\partial^{2} u_{i}}{\partial x_{j} \partial x_{j}}$,

where $u_{i}$ denotes the (instantaneous) velocity in the direction $\mathrm{x}_{i}, \rho$ represents the density, and $\mu$ is the kinematic viscosity. Equation (1) uses Einstein summation convention implying summation over terms indexed by $j$. With density and viscosity constant, the pressure gradient is related uniquely to the measured velocity field variables. The viscous terms on the right can be neglected at high Reynolds numbers as confirmed by several studies (e.g., van Oudheusden et al. 2007; Ghaemi et al. 2012). Although viscosity still plays a significant role in regions with high-velocity gradients, the length scales associated with viscous stresses are small and they therefore only make minor contributions during spatial integration of the pressure gradient. Although the viscous terms could still be maintained for completeness, they become easily corrupted in the presence of inaccuracies in the measured velocity data and will therefore generally be discarded in the ensuing analysis.

Considering now high-speed applications, apart from the increased challenge to obtain reliable measurement data as discussed earlier, a complication is to account for the unknown, variable density. In the previous studies (van Oudheusden et al. 2007; Souverein et al. 2007; van Oudheusden 2008), a convenient was found by removing the density as independent variable through the use of additional relations derived from the flow governing physical principles. In particular, the gas law $(\rho=p / R T)$ is used to replace the density in Eq. (1), while the adiabatic assumption of constant total temperature is employed to express temperature $(T)$ in terms of the velocity:

$T=T_{\infty}+\frac{V_{\infty}{ }^{2}-V^{2}}{2 c_{p}}=\frac{V_{\infty}{ }^{2}+\frac{\gamma-1}{2} M_{\infty}{ }^{2}\left(V_{\infty}{ }^{2}-V^{2}\right)}{\gamma R M_{\infty}{ }^{2}}$,

where $C_{\mathrm{p}}$ is the specific heat at constant pressure, $V$ is the velocity magnitude, $\gamma$ is the ratio of specific heats, $R$ is the gas constant, $M$ denotes the Mach number, and the subscript $\infty$ refers to freestream conditions.

A justification of this approach has been provided by an assessment of a synthetic PIV experiment constructed on the basis of a numerical simulation (van Gent et al. 2017a), which demonstrated that instantaneous pressure data could be reliably reconstructed under the assumptions mentioned above. However, as mentioned, the current interest is to obtain a reliable result for the mean pressure using velocity statistics in a Reynolds-averaging approach. The procedure was developed in an earlier publication (van Oudheusden 2008) and relies on the moderate-Mach-number assumption that density fluctuations can be neglected. This assumption is partly motived by physical arguments, reflecting Morkovin's hypothesis (Smits and Dussauge 2006) and partly by practical considerations, as density fluctuations cannot be obtained from the PIV measurement.

The specific objective of the present communication is to address the accuracy of this assumption, and the consistency of its implementation, by means of an evaluation of the relevance of all terms resulting from the Reynolds-averaging, including those that are discarded. The assessment methodology involves a theoretical order-of-magnitude analysis (Sect. 2) 
that is complemented with the results of a synthetic and a real PIV experiment (Sects. 3 to 5). The flow configuration of the experiments is an axisymmetric base flow at Mach 0.7, similar to the one studied in Blinde et al. (2015) and van Gent et al. (2017a). The compressible base flow is characterised by largescale unsteady separation and a richness in terms of flow scales making it particularly suited to provide a realistic and challenging test case. In addition, since it has been observed that base flows display less intense unsteadiness under supersonic conditions [e.g., Humble et al. (2007)], the transonic case is likely to pose a more critical combination of unsteadiness and compressibility.

\section{Theory}

\subsection{Governing relations to determine mean pressure in compressible flows}

The primary relation to calculate the mean pressure for unsteady compressible flows is given by the Reynolds-averaged momentum equation:

$\frac{\partial \bar{p}}{\partial x_{i}}=-\frac{\partial \overline{\rho u_{i} u_{j}}}{\partial x_{j}}$

where the momentum equation is written in conservative form to facilitate the Reynolds-averaging procedure. When needed, (part of) the momentum equation can be expressed in a non-conservative form by use of the continuity equation:

$\frac{\partial \overline{\rho u}_{j}}{\partial x_{j}}=0$

In the ensuing Reynolds-decomposition approach, variables are written as the sum of a mean and fluctuating component, denoted by an overbar and prime, respectively, e.g., $u=\bar{u}+u^{\prime}$. Depending on the specific conditions or approximation, fluctuations may be discarded for all variables (for a steady flow) or for the density only (as approximation). The resulting expressions correspond to those reported previously in van Oudheusden et al. (2007) and van Oudheusden (2008), respectively, but are repeated here for completeness.

\subsubsection{Steady-flow conditions}

Under the assumption that the flow is steady, all fluctuations can be discarded which simplifies the Reynolds-averaging operator in the continuity and momentum equations, such that the latter can be written as follows:

$\frac{\partial \bar{p}}{\partial x_{i}}=-\frac{\partial \bar{\rho} \bar{u}_{i} \bar{u}_{j}}{\partial x_{j}}=-\bar{\rho} \bar{u}_{j} \frac{\partial \bar{u}_{i}}{\partial x_{j}}$
This expression is similar to what would have been obtained for an incompressible flow, the difference, however, being that now in Eq. (5), the density is a spatial variable. To enable the computation of the pressure gradient from the velocity data (Souverein et al. 2007), the density is eliminated by means of the gas law $(\rho=p / R T)$, yielding:

$\frac{1}{\bar{p}} \frac{\partial \bar{p}}{\partial x_{i}}=\frac{\partial \ln \left(\bar{p} / p_{\infty}\right)}{\partial x_{i}}=-\frac{1}{R T} \overline{u_{j}} \frac{\partial \overline{u_{i}}}{\partial \overline{x_{j}}}$,

where the temperature $T$ is calculated from the local measured velocity using the adiabatic flow condition, Eq. (2).

\subsubsection{Relations for unsteady flow (neglecting density fluctuations)}

Considering an unsteady flow and incorporating the velocity fluctuations in the Reynolds-averaging, but neglecting the effect of density fluctuations (for moderate-Mach numbers), the pressure gradient follows from Eq. (3) as:

$\frac{\partial \bar{p}}{\partial x_{i}}=-\frac{\partial \bar{\rho} \overline{u_{i} u_{j}}}{\partial x_{j}}=-\bar{\rho} \bar{u}_{j} \frac{\partial \overline{u_{i}}}{\partial x_{j}}-\bar{\rho} \frac{\partial \overline{u_{i}^{\prime} u_{j}^{\prime}}}{\partial x_{j}}-\overline{u_{i}^{\prime} u_{j}^{\prime}} \frac{\partial \bar{\rho}}{\partial x_{j}}$.

The effect of flow compressibility is reflected in the variable value of the density in the first two terms on the right-hand side, and the presence of an additional third term, which is related to the spatial gradient of the mean density. Again, elimination of the density allows an explicit relation for the pressure gradient to be obtained:

$\left(\delta_{i j}+\frac{\overline{u_{i}^{\prime} u_{j}^{\prime}}}{R T}\right) \frac{\partial \ln \left(\bar{p} / p_{\infty}\right)}{\partial x_{j}}=-\frac{1}{R T}\left(\bar{u}_{j} \frac{\partial \overline{u_{i}}}{\partial x_{j}}+\frac{\partial \overline{u_{i}^{\prime}{ }_{i}^{\prime}}{ }_{j}}{\partial x_{j}}-\frac{\overline{u_{i}^{\prime}{ }_{i}^{\prime}}{ }_{j}}{T} \frac{\partial T}{\partial x_{j}}\right)$.

This relation was derived in van Oudheusden and Souverein (2007) and applied to an oblique shock-wave boundary-layer interaction at a freestream Mach number of 1.7, revealing the contribution of the turbulent fluctuations in the pressure determination to be minor in that case.

\subsubsection{Relations for unsteady flow (including density fluctuations)}

A proper evaluation that includes all variables in the Reynolds-averaging, i.e., both velocity and density fluctuations, yields:

$$
\begin{aligned}
\frac{\partial \bar{p}}{\partial x_{i}}= & -\frac{\partial \overline{\rho u_{i} u_{j}}}{\partial x_{j}}=-\bar{\rho} \overline{u_{i}} \frac{\partial \overline{u_{i}}}{\partial x_{j}}-\bar{\rho} \frac{\partial \overline{u_{i}^{\prime} u_{j}^{\prime}}}{\partial x_{j}}-\overline{u_{i}^{\prime} u_{j}^{\prime}} \frac{\partial \bar{p}}{\partial x_{j}} \\
& -\overline{\rho^{\prime} u_{j}^{\prime}} \frac{\partial \overline{u_{i}}}{\partial x_{j}}-\overline{u_{j}} \frac{\partial \overline{\rho^{\prime} u_{i}^{\prime}}}{\partial x_{j}}-\overline{\rho^{\prime} u_{i}^{\prime}} \frac{\partial \overline{u_{j}}}{\partial x_{j}}-\frac{\partial \overline{\rho^{\prime} u_{i}^{\prime} u_{j}^{\prime}}}{\partial x_{j}}
\end{aligned}
$$


As can be observed, in addition to the contributions already identified in Eq. (7), four additional terms appear that are related to the density fluctuations. Since the density fluctuations themselves cannot easily be accessed from the velocity measurement, either directly or indirectly through the additional governing equations, no attempt can be made to incorporate these in the final pressure determination, as was done in the two previous sections. Instead, the purpose of this formulation is to provide the framework for the following theoretical and numerical analysis.

\subsection{Order-of-magnitude analysis}

To perform an order-of-magnitude analysis of the different contributions to the pressure gradient (Eq. 9), appropriate scaling values (indicated by an asterisk) are introduced for the different parameters involved, such that:

$\bar{\rho} \sim \rho^{*} ; \bar{u}_{i} \sim U^{*} ; u_{i}^{\prime} \sim u^{*} ; \frac{\partial}{\partial x_{j}} \sim \frac{1}{L^{*}}$.

To find the appropriate scaling for the temporal and spatial density variations, $\rho^{\prime}$ and $\partial \bar{\rho} / \partial x_{j}$, the gas law $(\rho=p / R T)$ is cast in differential form:

$\mathrm{d} \rho=\frac{1}{R T} \mathrm{~d} \rho-\frac{\rho}{T} \mathrm{~d} T$.

In view of the momentum equation: $\mathrm{d} p \sim \rho V \mathrm{~d} V$ and with the assumption of constant total temperature: $c_{\mathrm{p}} \mathrm{d} T \sim V \mathrm{~d} V$, this can be written as follows:

$\mathrm{d} \rho \sim \rho M^{2} \frac{\mathrm{d} V}{V}$.

Interpreting $\mathrm{d} \rho$ as either a temporal or spatial variation (i.e., fluctuation or gradient) of the density, the following results are obtained:

$\rho^{\prime} \sim \rho M^{2} \frac{V^{\prime}}{V} \sim \rho^{*} M^{2} \frac{u^{*}}{U^{*}}$

$\frac{\partial \bar{\rho}}{\partial x_{j}} \sim \rho M^{2} \frac{1}{V} \frac{\partial V}{\partial x_{j}} \sim M^{2} \frac{\rho^{*}}{L}$.

Finally, although mean velocity gradients scale according to Eq. (10) as: $\partial \overline{u_{i}} / \partial x_{j} \sim U^{*} / L^{*}$, this is not appropriate for the divergence term $\partial \overline{u_{j}} / \partial x_{j}$ that occurs in Eq. (9), as it does not reflect that the divergence should vanish under incompressible flow conditions. Instead, upon invoking the continuity equation [Eq. (4)], we find:

$\frac{\partial \overline{u_{j}}}{\partial x_{j}} \approx-\frac{\overline{u_{j}}}{\bar{\rho}} \frac{\partial \bar{\rho}}{\partial x_{j}} \sim M^{2} \frac{U^{*}}{L^{*}}$.

Application of these estimates provides the order-of-magnitude estimate of each of the contributions to the pressure
Table 1 Order-of-magnitude analysis

\begin{tabular}{lll}
\hline $\begin{array}{l}\text { Contribution } \\
\text { to the pressure } \\
\text { gradient }\end{array}$ & Order-of-magnitude & $\begin{array}{l}\text { Order-of-magnitude } \\
\text { relative to mean flow } \\
\text { acceleration }\end{array}$ \\
\hline $\bar{\rho} \overline{u_{j}} \frac{\partial \bar{u}_{i}}{\partial x_{j}}$ & $\rho^{*} \frac{U^{* 2}}{L^{*}}$ & 1 \\
$\bar{\rho} \frac{\partial u_{i}^{\prime} u_{j}^{\prime}}{\partial x_{j}}$ & $\rho^{*} \frac{u^{* 2}}{L^{*}}$ & $\left(\frac{u^{*}}{U^{*}}\right)^{2}$ \\
$\overline{u_{i}^{\prime} u_{j}^{\prime}} \frac{\partial \bar{\rho}}{\partial x_{j}}$ & $\rho^{*} M^{2} \frac{u^{* 2}}{L^{*}}$ & $M^{2}\left(\frac{u^{*}}{U^{*}}\right)^{2}$ \\
$\overline{\rho^{\prime}} \overline{u_{j}^{\prime}} \frac{\partial \overline{u_{i}}}{\partial x_{j}}$ & $\rho^{*} M^{2} \frac{u^{* 2}}{L^{*}}$ & $M^{2}\left(\frac{u^{*}}{U^{*}}\right)^{2}$ \\
$\overline{u_{j}} \frac{\partial \rho^{\prime} u_{i}^{\prime}}{\partial x_{j}}$ & $\rho^{*} M^{2} \frac{u^{* 2}}{L^{*}}$ & $M^{2}\left(\frac{u^{*}}{U^{*}}\right)^{2}$ \\
$\overline{\rho^{\prime} u_{i}^{\prime}} \frac{\partial \bar{u}_{j}}{\partial x_{j}}$ & $\rho^{*} M^{4} \frac{u^{* 2}}{L^{*}}$ & $M^{4}\left(\frac{u^{*}}{U^{*}}\right)^{2}$ \\
$\overline{\frac{\partial \rho^{\prime} u_{i}^{\prime} u_{j}^{\prime}}{\partial x_{j}}}$ & $\rho^{*} M^{2} \frac{u^{* 3}}{L^{*}}$ & $M^{2}\left(\frac{u^{*}}{U^{*}}\right)^{3}$ \\
\hline
\end{tabular}

gradient in Eq. (9), which are collected in Table 1. Note that the first three rows indicate the contributions that are accessible from the PIV measurement and were proposed to be included in the evaluation of the pressure gradient by van Oudheusden (2008) [see Eq. (7)]. The first four rows in table 1 indicate all terms that involve density fluctuations and cannot be obtained from the PIV measurements.

Observing the results for the different terms, it is concluded that all terms, apart from the mean flow acceleration, scale in proportion to $u^{*} / U^{*}$ and the density fluctuation terms all involve an additional scaling with the Mach number. However, it is interesting to observe that the third term, maintained in Eq. (7), appears to be of similar order as the first two additional terms related to the density fluctuations. This would suggest that either its inclusion in Eq. (7) is unnecessary, or that the additional terms identified in Eq. (9) should not be neglected with respect to the terms maintained in Eq. (7). However, this is only an order-ofmagnitude estimate and a proper evaluation of their relative importance should be based on a quantitative assessment, as undertaken in the following section.

\section{Numerical assessment}

The theory from Sect. 2 is assessed in a series of assessments. This first assessment employs the result of a zonal detached eddy simulation (ZDES) [e.g., Weiss et al. (2009)], which was specifically designed for the purpose of testing pressure reconstruction techniques (van Gent et al. 2017a). The simulation was set up to represent the same geometry and flow conditions as those of the experimental assessment considered in Sect. 5. 


\subsection{Description of the test case}

Figure 1 depicts the model geometry and the measurement domain for the simulated PIV experiment (Sect. 4). The model main body has a diameter $(D)$ of $50 \mathrm{~mm}$ and the afterbody has a diameter of $20 \mathrm{~mm}(0.4 \mathrm{D})$, resulting in a step height of $15 \mathrm{~mm}(0.3 \mathrm{D})$. Wind tunnel walls were omitted in the simulation for simplicity and robustness. The free stream flow has a total pressure $\left(p_{\mathrm{t}}\right)$ of $200 \mathrm{kPa}$ and a total temperature $\left(T_{\mathrm{t}}\right)$ of $285 \mathrm{~K}$, corresponding to a free stream pressure of $144,186 \mathrm{~Pa}$, a free stream temperature of $260 \mathrm{~K}$, and a free stream velocity $\left(U_{\infty}\right)$ of $226 \mathrm{~m} \mathrm{~s}^{-1}$. The Reynolds number based on the model main body diameter $\left(\operatorname{Re}_{\mathrm{D}}\right)$ is 1.3 million.

The present analysis focuses on a region directly downstream of the step with a size of $1.2 \mathrm{D} \times 0.47 \mathrm{D} \times 0.08 \mathrm{D}$ $(60 \mathrm{~mm} \times 24 \mathrm{~mm} \times 4 \mathrm{~mm}, L \times H \times W)$ that corresponds with the measurement domain of the simulated experiment (see Sect. 4). To facilitate the computation of spatial gradients, the original simulation data are interpolated onto a rectangular grid of $171 \times 67 \times 11$ point in $\mathrm{x}-, \mathrm{y}-$, and $\mathrm{z}$-directions, using natural neighbour interpolation (Sibson 1981). The data set consists of 5000 snapshots with a time separation of $2 \mu \mathrm{s}$, covering a total duration of $10 \mathrm{~ms}$. The reader is referred to van Gent et al. (2017a) for further details.

To characterise the mean properties and fluctuations in the flow, Fig. 2 shows the mean streamwise velocity, mean pressure, and mean density (left figures) as well as the turbulence intensity (T.I.) and the root mean square of pressure and density fluctuations with respect to their mean value (right figures). Contours are shown for the centre plane of the region of interest. The pressure coefficient $\left(C_{\mathrm{p}}\right)$ and the turbulence intensity (T.I.) are defined as follows: $C_{\mathrm{p}}=\left(p-p_{\infty}\right) /\left(\frac{1}{2} \gamma p_{\infty} M_{\infty}^{2}\right)$ and T.I. $=\sqrt{\left(\sigma_{\mathrm{u}}^{2}+\sigma_{\mathrm{v}}^{2}+\sigma_{\mathrm{w}}^{2}\right) / 3}$, where $\sigma_{\mathrm{u}}^{2}, \sigma_{\mathrm{v}}^{2}$, and $\sigma_{\mathrm{w}}^{2}$ denote the variances of the velocity components in $x_{-}^{-}, y_{-}$, and z-directions, respectively.

The mean flow field displays a shear layer that emanates from the corner of the step at $y / D=0.3$ and grows in downstream direction towards reattachment on the afterbody at about $x / D=1.2$. Below the shear layer lies a large-scale separated region where the mean velocity, pressure, and density reach minimum values of about $\bar{u} \approx-0.35 U_{\infty}, C_{\mathrm{p}} \approx-0.24$, and $\rho \approx 0.86 \rho_{\infty}$ Velocity, pressure, and density increase towards reattachment. Elevated levels of turbulence intensity, pressure, and density fluctuations are notably present in the shear layer and the reattachment region (right figures).

\subsection{Pressure gradient evaluation}

The data of the numerical simulation allow to directly evaluate the different terms that make up the mean pressure gradient [Eq. (9)]; these are defined as the partial contributions $(\nabla \bar{p})_{i}$ to the total pressure gradient, in the sense that $\nabla \bar{p}=\sum_{i}(\nabla \bar{p})_{i}$. Figure 3 shows the spatial distributions of the total pressure gradient (top figures) and of the contributions of different terms. The left column corresponds to the pressure gradient in $\mathrm{x}$-direction $(\partial \bar{p} / \partial x)$ and right column to the gradient in y-direction $(\partial \bar{p} / \partial y)$. All pressure gradients have been non-dimensionalised according to $C_{\nabla \bar{p}}=D \nabla \bar{p} / \frac{1}{2} \gamma p_{\infty} M_{\infty}^{2}$. Noting the difference in the colour scale, the figure shows that the mean flow terms (figures c, d) and Reynolds-stresses terms (figures e, f) are the dominant contributors to the total pressure gradient (figures a, b) and are an order-of-magnitude greater than the other terms. The mean flow terms are the only terms that substantially contribute to the pressure gradient in the recirculation region and in the freestream where overall fluctuations in the flow are small (compare Fig. 2). The contributions from the Reynolds-stresses are particularly large in the shear layer and the reattachment

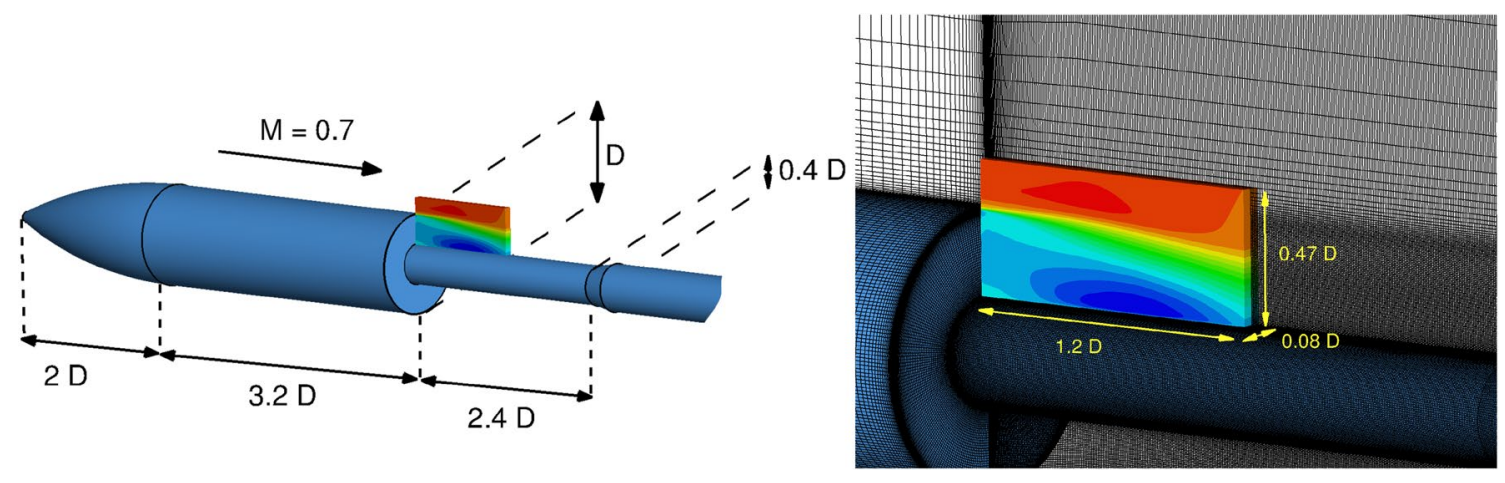

Fig. 1 Test geometry: overview and detail of the numerical mesh in the base-flow region ( $D$ is the main body diameter); the coloured insert indicates the measurement domain for the simulated experi- ment; filled colour contours depict the mean streamwise velocity [reproduced with permission from van Gent et al. (2017a)] 

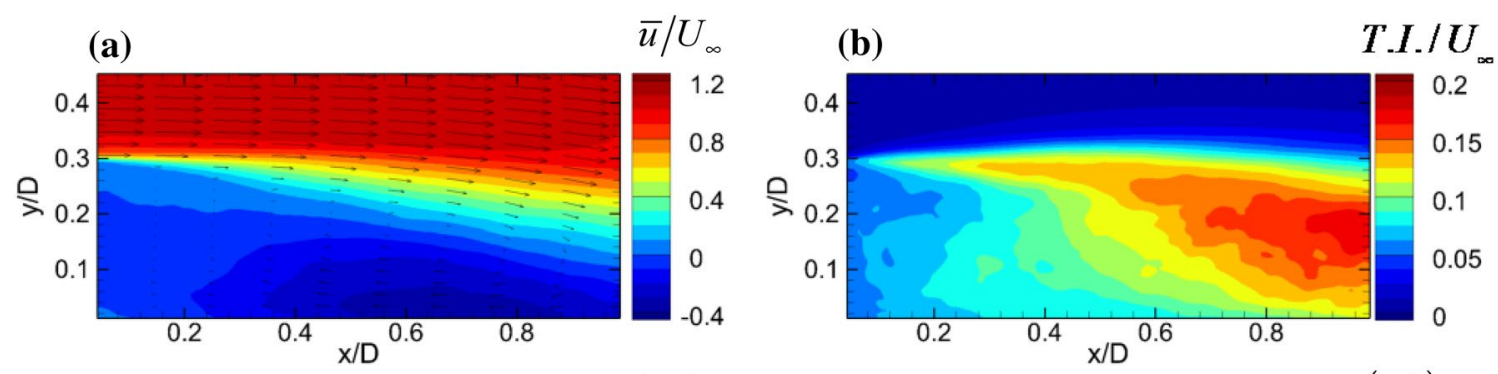

(c)



(e)

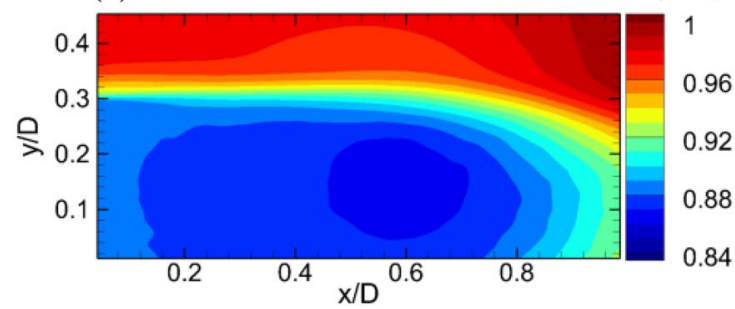

$\bar{\rho} / \rho_{\infty}$

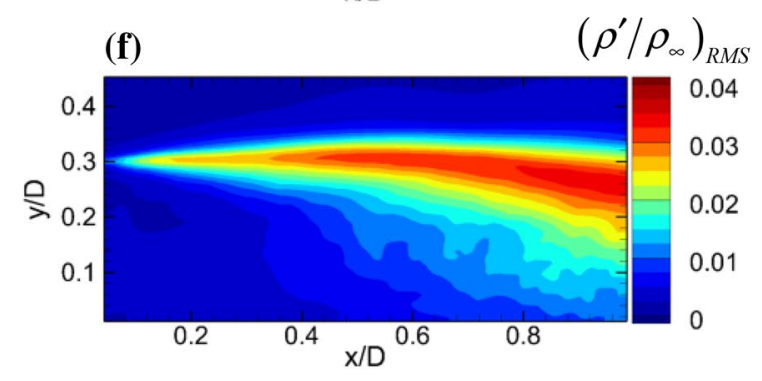

Fig. 2 Characterization of the flow field statistics: a mean streamwise velocity, b turbulence intensity, c mean pressure, d pressure fluctuation levels, e mean density, and $\mathbf{f}$ density fluctuations [partly adapted from van Gent et al. (2017a)]

region. Interestingly, looking at the pressure gradient in streamwise direction (left column), the contributions of the mean flow and Reynolds-stresses terms are large in the shear layer, but cancel each other out. The general behaviour of the pressure gradient in the region of the shear layer, regarding the contributions of the mean flow (figures c, d), Reynolds-stresses (figures e, f), and density gradient (figures $\mathrm{g}, \mathrm{h}$ ), is in good qualitative agreement with theory from Sect. 2.

The relevance of each pressure gradient term is further assessed by means of an integral measure that represents the average value of the norm of the contribution:

$\left\langle\left\|\left(C_{\nabla \bar{p}}\right)_{i}\right\|\right\rangle=\frac{1}{A} \iiint\left\|\left(C_{\nabla \bar{p}}\right)_{i}\right\| \mathrm{d} A$,

where $A$ is the area of interest, which is taken to correspond to the measurement volume of the simulated experiment. Table 2 provides an overview of the values of this integral measure for the different terms. Note that as the integral measure is defined from the norm of the contribution, the values of all contributions do not add up to exactly the same value as that for the total pressure gradient itself. The table confirms that the contributions from the mean flow and the Reynolds-stresses terms are, indeed, larger than those of the other terms (by about two orders-of-magnitude), the latter representing not more than 0.02 of the measure for the total pressure gradient.

\section{Simulated experimental assessment}

The numerical simulation from Sect. 3 has been used to construct a simulated tomographic PIV experiment. The specific purpose of this assessment, in addition to the one from the previous section, is to model the issues involved in a "real" PIV experiment, yet having the reference pressure field available for validation. These additional issues are, notably: (1) the finite resolution and accuracy of the PIV measurement, and (2) the additional modelling steps required to extract the pressure from the velocity fields, as discussed in the introduction. 
(a)

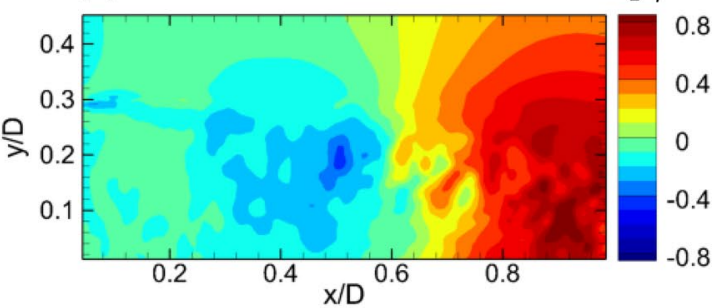

$-\bar{\rho} \overline{u_{j}}\left(\partial \bar{u} / \partial x_{j}\right)$



(e)

$-\bar{\rho}\left(\partial \overline{u_{j}{ }^{\prime} u^{\prime}} / \partial x_{j}\right)$

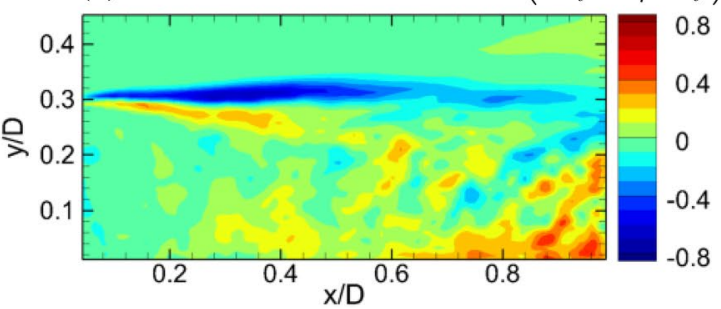

(g)

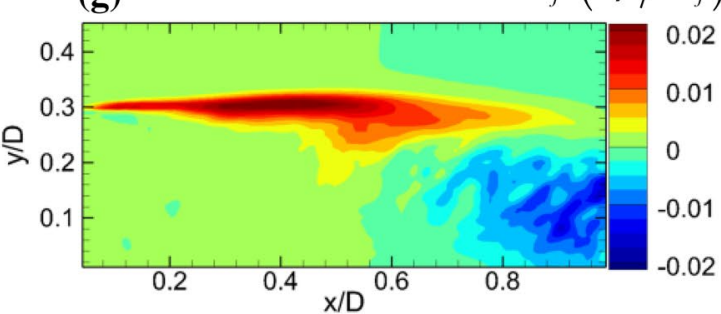

(i)

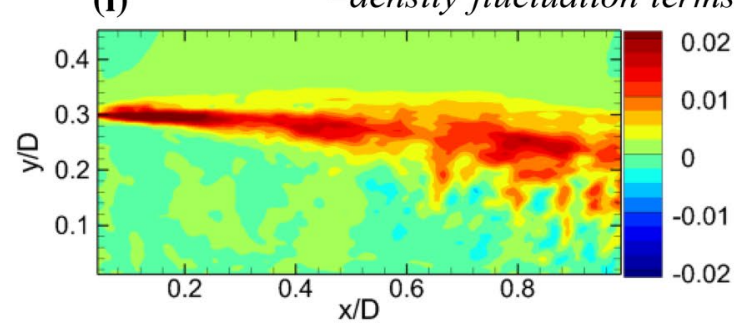

(b)

$\partial \bar{p} / \partial y$

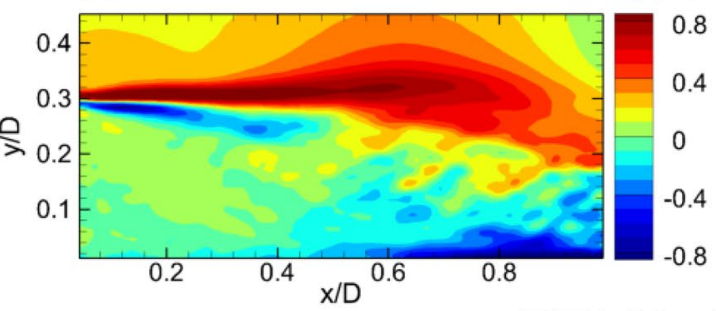

(d)

$-\bar{\rho} \overline{u_{j}}\left(\partial \bar{v} / \partial x_{j}\right)$
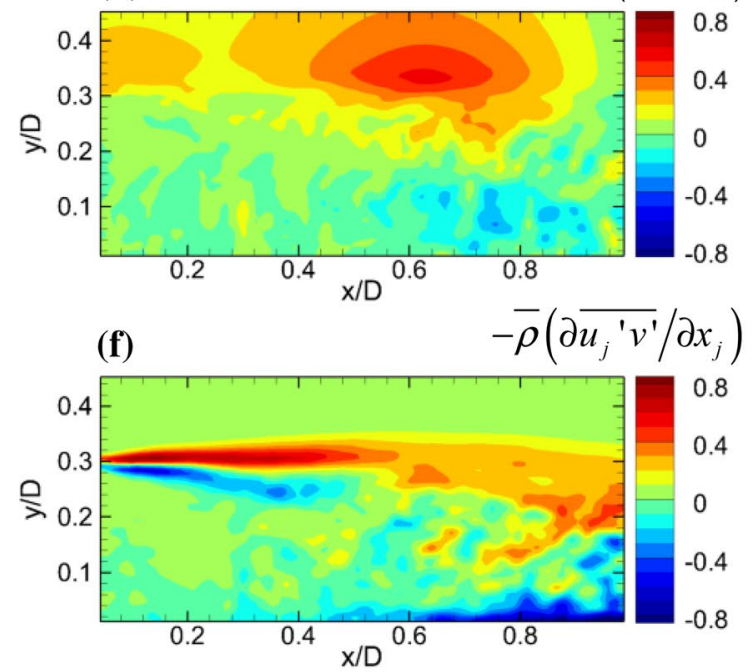

(h)

$-\overline{v^{\prime} u_{j}{ }^{\prime}}\left(\partial \bar{\rho} / \partial x_{j}\right)$
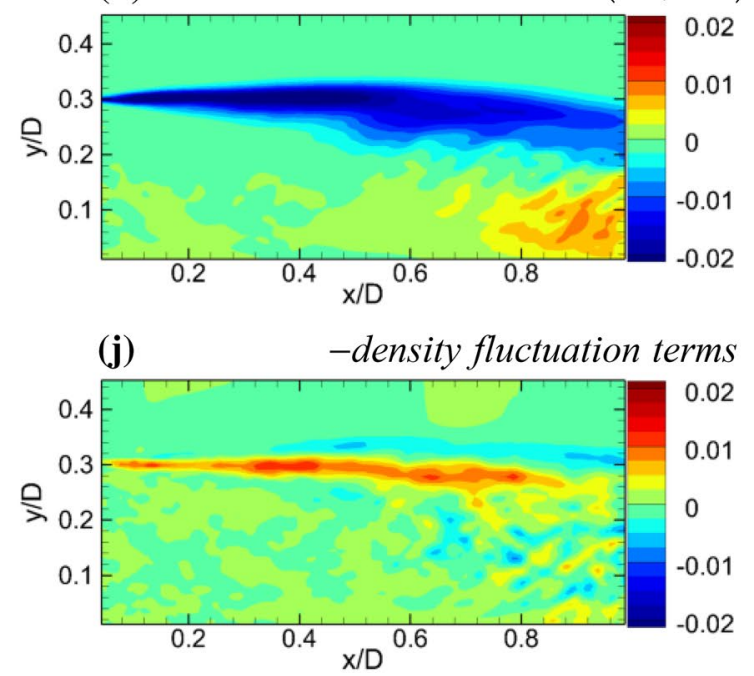

Fig. 3 Contributions to the pressure gradient: $\mathbf{a}, \mathbf{b}$ total pressure gradient, $\mathbf{c}, \mathbf{d}$ mean flow terms, $\mathbf{e}, \mathbf{f}$ Reynolds-stresses terms, $\mathbf{g}, \mathbf{h}$ density-gradient terms and $\mathbf{i}, \mathbf{j}$ density fluctuation terms; left column: contributions to $\partial \bar{p} / \partial x$; right column: contributions to $\partial \bar{p} / \partial y$

\subsection{Simulation of PIV experiment}

Imaginary seeding particles are traced through the simulated flow field that is represented by the numerical data. Particle images are then obtained by projecting the three-dimensional particle positions onto two-dimensional sensors. The digital resolution of the images is 22.9 vox $\mathrm{mm}^{-1}$ (optical magnification $M=0.12$ ). The virtual cameras are calibrated by a combination of geometric calibration and volume selfcalibration (Wieneke 2008). Noise is added to the synthetic particle images and all processing steps associated with tomographic PIV are subsequently applied to generate a 
Table 2 Relative importance of the contributions to the pressure gradient

\begin{tabular}{lll}
\hline $\begin{array}{l}\text { Contribution } \\
\text { to the pressure } \\
\text { gradient }\end{array}$ & $\begin{array}{l}\text { Integral measure } \\
\left\langle\left\|\left(C_{\nabla \bar{p}}\right)_{i}\right\|\right\rangle\end{array}$ & $\begin{array}{l}\text { Integral measure relative } \\
\text { to that of the total pressure } \\
\text { gradient }\end{array}$ \\
\hline $\begin{array}{l}\text { Total pressure } \\
\text { gradient } \frac{\partial \bar{p}}{\partial x_{j}}\end{array}$ & $4.2 \times 10^{-1}$ & 1 \\
$\bar{\rho} \bar{u}_{j} \frac{\partial \bar{u}_{i}}{\partial x_{j}}$ & $3.6 \times 10^{-1}$ & 0.86 \\
$\bar{\rho} \frac{\partial \overline{u_{i}^{\prime} u_{j}^{\prime}}}{\partial x_{j}}$ & $1.8 \times 10^{-1}$ & 0.43 \\
$\overline{u_{i}^{\prime} u_{j}^{\prime}} \frac{\partial \bar{\rho}}{\partial x_{j}}$ & $4.1 \times 10^{-3}$ & $<0.01$ \\
$\overline{\rho^{\prime} u_{j}^{\prime}} \frac{\partial u_{i}^{\prime}}{\partial x_{j}}$ & $3.1 \times 10^{-3}$ & $<0.01$ \\
$\overline{u_{j}} \frac{\partial \rho^{\prime} u_{i}^{\prime}}{\partial x_{j}}$ & $2.4 \times 10^{-3}$ & $<0.01$ \\
$\overline{\rho^{\prime} u_{i}^{\prime}} \frac{\partial u_{j}^{\prime}}{\partial x_{j}}$ & $6.4 \times 10^{-5}$ & $<0.01$ \\
$\frac{\partial \rho^{\prime} u_{i}^{\prime} u_{j}^{\prime}}{\partial x_{j}}$ & $3.1 \times 10^{-3}$ & $<0.01$ \\
\hline
\end{tabular}

data set representative of realistic PIV results, albeit under optimum imaging conditions. The resulting PIV data consist of $171 \times 67 \times 11$ velocity vectors in $\mathrm{x}-$, $\mathrm{y}$-, and $\mathrm{z}$-directions. For more details on the simulated experiment, the reader is referred to van Gent et al. (2017a).

\subsection{Pressure results}

The velocity fields are used to calculate the pressure gradient according to the logarithmic formulation of Eqs. (6) and (8). The problem is discretized using a similar discretisation as Jeon et al. (2015) and the resulting system of linear equations is solved via the use of QR decomposition. Pressure gradients are used as Neumann boundary conditions on all sides of the domain except for the top surface, where reference pressure as obtained from the simulation is prescribed as Dirichlet boundary condition.

Figure 4 shows the reference pressure field from the simulation data (top figure), the pressure fields reconstructed from the PIV data (left figures) as well as their respective difference (right figures). Comparison of Fig. 4a, b shows that reconstruction of pressure fields from mean flow terms only [Eq. (6)] leads to substantial error levels (also see figure c). Figure $4 d$ shows that the additional inclusion of Reynolds-stresses terms leads to a dramatic improvement (compare figure $\mathrm{c}$ and e). Further addition of the densitygradient terms (Fig. 4f) has a little effect, if not leading to slightly higher errors. Only slightly more accurate results could be achieved using the reference temperature from the simulation data instead of the temperature calculated by assuming adiabatic flow [Eq. (2)].

To quantify these findings, Table 3 shows the r.m.s. value of the error fields depicted in the right figures. As indicated in the table, the normalized error is $17 \%$ when only the mean flow terms are used and reduces to about $5 \%$ when including the Reynolds-stresses terms.

As surface pressure is of great importance for many applications, particular attention is given to the reconstruction of surface pressure from the PIV results. Figure 5a shows the mean pressure distribution along the bottom edge of the measurement domain. Similarly, Fig. 5b compares the mean pressure profiles for $x / D=0.6$ where the pressure attains its minimum value. Reference data have been obtained by interpolating the simulation data to the PIV grid. Other pressure profiles have been reconstructed from velocity data using different terms. The figures also include a profile obtained using reference density data instead of assuming adiabatic flow to show the impact using an adiabatic flow model as well as a profile reconstructed from reference velocity data to show the impact of measurement errors from PIV and its spatial filtering behaviour.

Confirming earlier findings, the figures show that including the Reynolds-stresses terms in the pressure reconstruction leads to a notably improvement over using only the mean flow terms. Inclusion of the density-gradient terms has no perceivable impact. Using reference density data and, therefore, avoiding the need to assume adiabatic flow only leads to a minor improvement, again confirming the validity of the adiabatic flow model. Figure 5b shows that using reference velocity data leads to a step improvement compared to using PIV velocity data. This is attributed to the spatial filtering behaviour of PIV. Regardless, Fig. 5a shows that the surface pressure can be accurately reconstructed from PIV measurements.

\section{Actual experimental assessment}

A final assessment of the mean pressure reconstruction procedure uses data from an actual PIV experiment. Validation is performed by comparison to additional measurements by pressure transducers.

\subsection{Experimental arrangement}

The experiments have been performed in the transonicsupersonic wind tunnel (TST-27) at the Aerodynamics Laboratory at Delft University of Technology. The facility has a test section of $280 \mathrm{~mm}$ (width) $\times 270 \mathrm{~mm}$ (height). In the present experiments, the wind tunnel is operated at a total pressure $\left(p_{0}\right)$ of $2.0 \times 10^{5} \mathrm{~Pa}$. Total temperature $\left(T_{0}\right)$ is approximately $280 \mathrm{~K}$. The nominal Mach number 


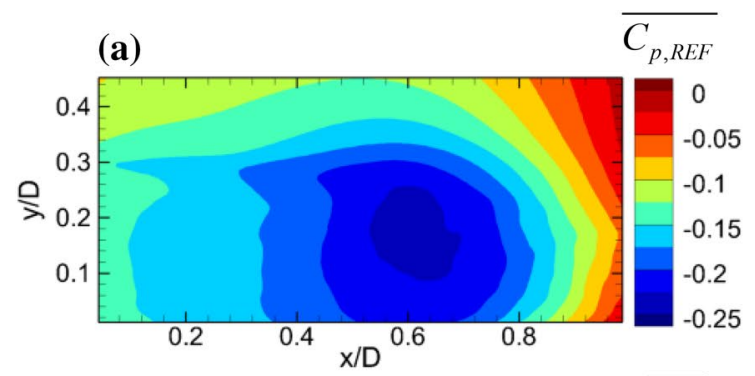

(b)

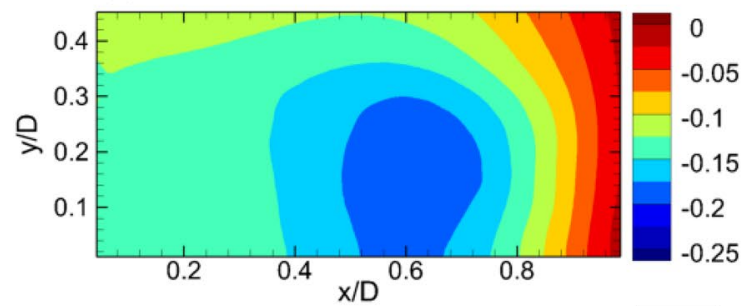

(d)

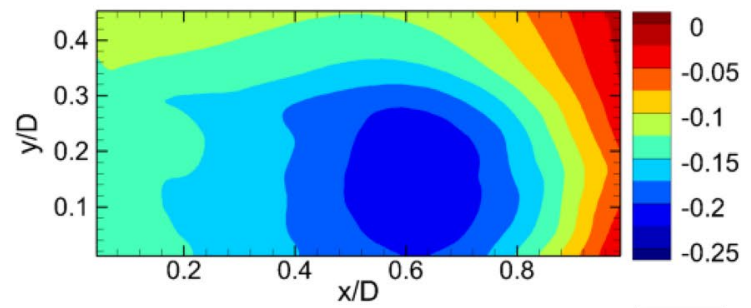

(f)

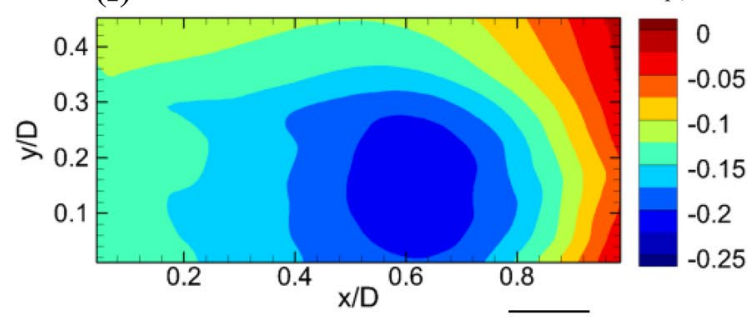

(h)

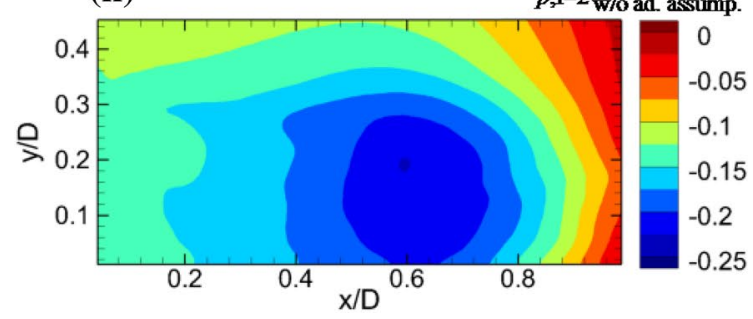

Fig. 4 Mean pressure: a reference pressure field, b pressure field reconstructed using mean flow terms and $\mathbf{c}$ corresponding error, $\mathbf{d}$ pressure field reconstructed using mean flow and Reynolds-stresses terms and e corresponding error, $\mathbf{f}$ pressure field reconstructed using

is set at 0.7 . Based on pressure static pressure measurements in the test section $\left(p_{\infty}=1.38 \times 10^{5} \mathrm{~Pa}\right)$ and assuming isentropic expansion, the effective free stream Mach number $\left(M_{\infty}\right)$ is determined to be 0.75 . The difference
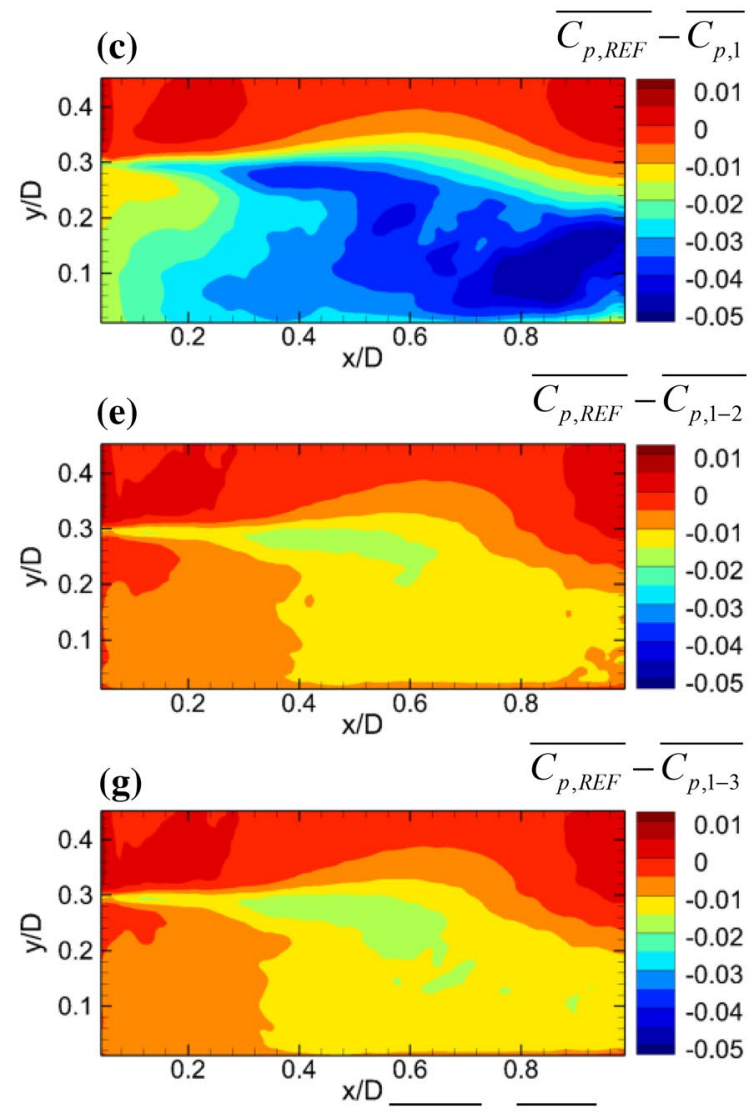

(i)
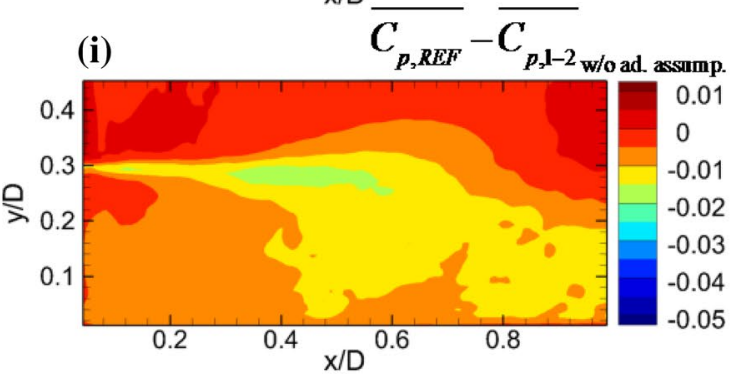

mean flow, Reynolds-stresses and density-gradient terms and $\mathbf{g}$ corresponding error, $\mathbf{h}$ pressure field reconstructed using mean flow and Reynolds-stresses w/o adiabatic assumption, and $\mathbf{i}$ corresponding error

with the nominal Mach number is caused by the reduction in the effective cross-sectional area due to the presence of the model. The freestream velocity $\left(U_{\infty}\right)$ is $238 \mathrm{~m} \mathrm{~s}^{-1}$ 
Table 3 Error assessment of reconstructed pressure fields

\begin{tabular}{lll}
\hline Basis for pressure field reconstruction & $\left(\overline{C_{\mathrm{p}, \mathrm{REF}}}-\overline{C_{\mathrm{p}}}\right)_{\mathrm{RMS}}$ & $\frac{\left(\overline{\mathrm{C}_{\mathrm{p}, \mathrm{REF}}}-\overline{\mathrm{C}_{\mathrm{p}}}\right)_{\mathrm{RMS}}}{\left(\overline{C_{\mathrm{p}, \mathrm{REF}}}\right)_{\mathrm{RMS}}}$ \\
\hline Mean flow terms only & $2.48 \times 10^{-2}$ & 0.174 \\
Mean flow + Reynolds-stresses terms & $0.77 \times 10^{-2}$ & 0.054 \\
Mean flow + Reynolds-stresses terms (without adiabatic assumption) & $0.74 \times 10^{-2}$ & 0.052 \\
Mean flow + Reynolds-stresses + density-gradient terms & $0.86 \times 10^{-2}$ & 0.060 \\
$\begin{array}{l}\text { Mean flow + Reynolds-stresses + density-gradient terms (without adi- } \\
\text { abatic assumption) }\end{array}$ & $0.80 \times 10^{-2}$ & 0.056 \\
\hline
\end{tabular}

(a)

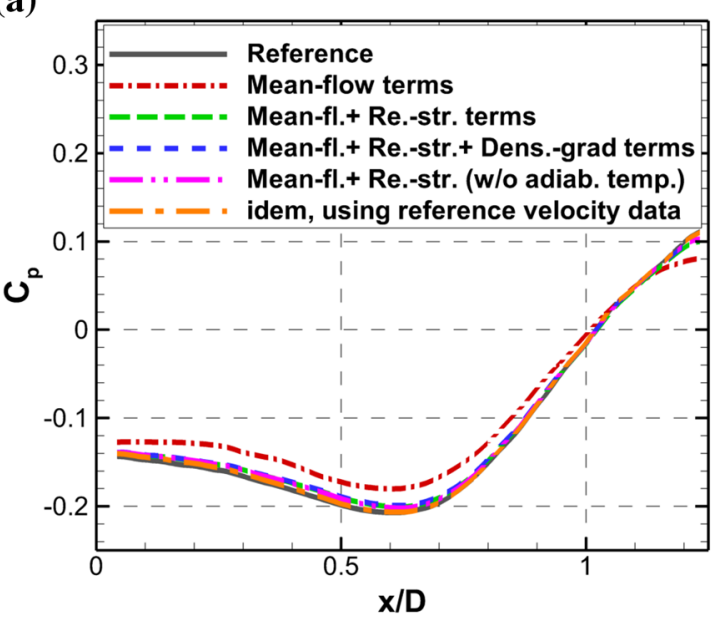

(b)

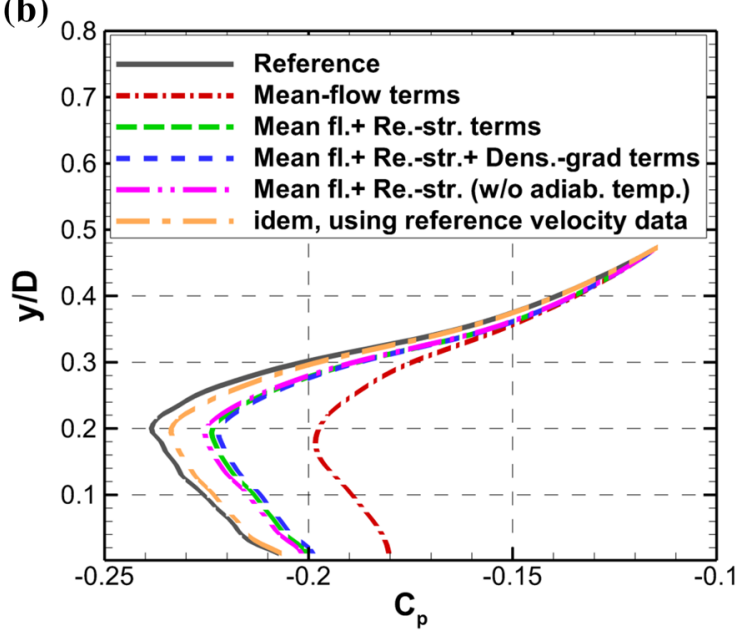

Fig. 5 a Mean pressure profile over afterbody located at the bottom edge of the PIV measurement domain and $\mathbf{b}$ for $x / D=0.6$

and the Reynolds number based on the model diameter $\left(\operatorname{Re}_{\mathrm{D}}\right)$ is $1.3 \times 10^{6}$.

The wind tunnel model has a similar geometry as the model used in the simulation (see Fig. 1). The main body is held from the rear by a sting that also acts as the afterbody.

The PIV measurements were performed in a thin volume located downstream of the step over the afterbody surface. The size of the measurement volume is $1.7 \mathrm{D} \times 1.0 \mathrm{D} \times 0.1 \mathrm{D}$ $(85 \mathrm{~mm} \times 50 \mathrm{~mm} \times 5 \mathrm{~mm}, L \times H \times W$ ). The flow was seeded with Di-Ethyl-Hexyl-Sebacat (DEHS) particles. The particles have a nominal diameter of $1 \mu \mathrm{m}$ and a response time of about $2 \mu$ s (Ragni et al. 2010). The particles were introduced into the settling chamber via a seeding rake connected to a PIVTEC atomizing DEHS seeder. Based on visual inspection of various regions in different images, the particle density is estimated to vary between 0.03 and 0.07 particles per pixel (ppp).

Illumination was provided by double-cavity $\mathrm{Nd}-\mathrm{Yag}$ lasers. The laser light was introduced into the wind tunnel by an optical probe that was located downstream of the test section. The laser light was shaped into a sheet using optics inside the probe. Upon exiting the probe, the edges of the laser light sheets were cut off to obtain a more clearly defined laser sheet and to achieve a light sheet thickness of approximately $5 \mathrm{~mm}$. The synchronization of all components and the acquisition of image data were performed using an external timing unit. The time separation between two consecutive pulses was set at $2.5 \mu \mathrm{s}$, corresponding to a freestream particle displacement of 15 voxels in the reconstruction. Recording was performed by $5 \mathrm{LaVision}$ Imager LX 2MP cameras (pixel resolution $1628 \times 1236$ pixel, pixel size $4.4 \times 4.4$ micron) arranged, as shown in Fig. 6, at a recording rate of at $5 \mathrm{~Hz}$. Each camera was equipped with a $75 \mathrm{~mm}$ Tamron C-mount lens attached to a custommanufactured Scheimpflug mount. Their aperture was set at $\mathrm{f} / 5.6$, leading to a focal depth of about $9.6 \mathrm{~mm}(0.19 \mathrm{D})$. The digital resolution is 23.0 vox $\mathrm{mm}^{-1}$ (optical magnification $M=0.10)$.

Images are pre-processed by subtracting the minimum intensity over the time-series and subtracting the minimum intensity within 31 pixel-sized kernels. The intensity is normalized by the average intensity of the time-series. After volume self-calibration (Wieneke 2008), reconstructed objects are initialized by the multiplicative line-of-sight (MLOS) method (Atkinson and Soria 2009) and refined by eight iterations of the fast-MART algorithm in the LaVision 




(b)

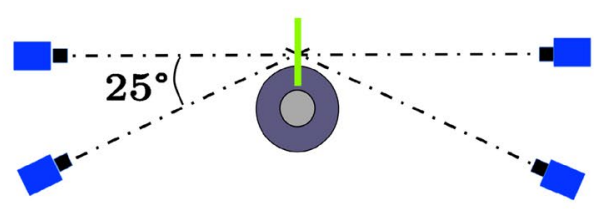

Fig. 6 Schematics of camera setup; a top view; and $\mathbf{b}$ back view; figures do not show proper relative dimensions

8.1 software. Gaussian smoothing is performed after each iteration, excluding the final iteration (Discetti et al. 2013). Each reconstructed voxel was required to be visible by least three cameras. Due to the use of five cameras with distinctly different viewing angles and a relatively thin volume, the signal-to-noise ratio of the resulting reconstruction exceeded ten, as inferred from its intensity distribution in z-direction (the 'z-profile').

Velocity data are obtained by direct cross-correlation of the reconstructed intensity fields using an iterative multigrid volume deformation (Scarano and Riethmuller 2000), symmetric block direct correlation (Discetti and Astarita 2012), and Gaussian window weighting. Vector fields from intermediate correlation steps are enhanced for the next iteration by removing spurious vectors, identified by universal outlier detection (Westerweel and Scarano 2005), replacing them using linear interpolation, and by Gaussian smoothing of the velocity field. The final three iterations are performed with an interrogation volume size of 32 voxels at $75 \%$ overlap. As for the intermediate steps, spurious vectors are removed after the final step by universal outlier detection and replaced using linear interpolation. The amount of removed vectors after the final correlation step is typically below $1 \%$. Based on the ensemble size of 500 images, the uncertainty in mean velocity is $(1 / 500)^{-1 / 2}=4.5 \%$ of the velocity fluctuations, which translates to a maximum of about $1 \%$ of the freestream velocity. The uncertainty of the Reynolds-stresses is about $(2 / 500)^{1 / 2}=6.3 \%$ (of the Reynolds-stresses) (Sciacchitano and Wieneke 2016).

\subsection{Flow characterization}

To characterise the flow field, Fig. 7 shows the mean streamwise velocity and turbulence intensity. The figures include the contours of the model. Note that a small flow region close to the rear surface of the model main body is obscured as a result of the camera viewing angles. Overall, the experimental data show a similar flow organisation as the simulation (compare Fig. 2), although mean flow reattachment occurs more upstream at $x / D=1.0$. Elevated turbulence levels near the top of the domain and close to the afterbody are attributed to measurement noise, since they do not appear in the simulation data and are accompanied by poorer illumination and seeding conditions than what is typical for other parts of the measurement domain.

By performing additional numerical simulations, it was checked that the longer reattachment length in the simulation is not due to the confinement effect of the wind tunnel
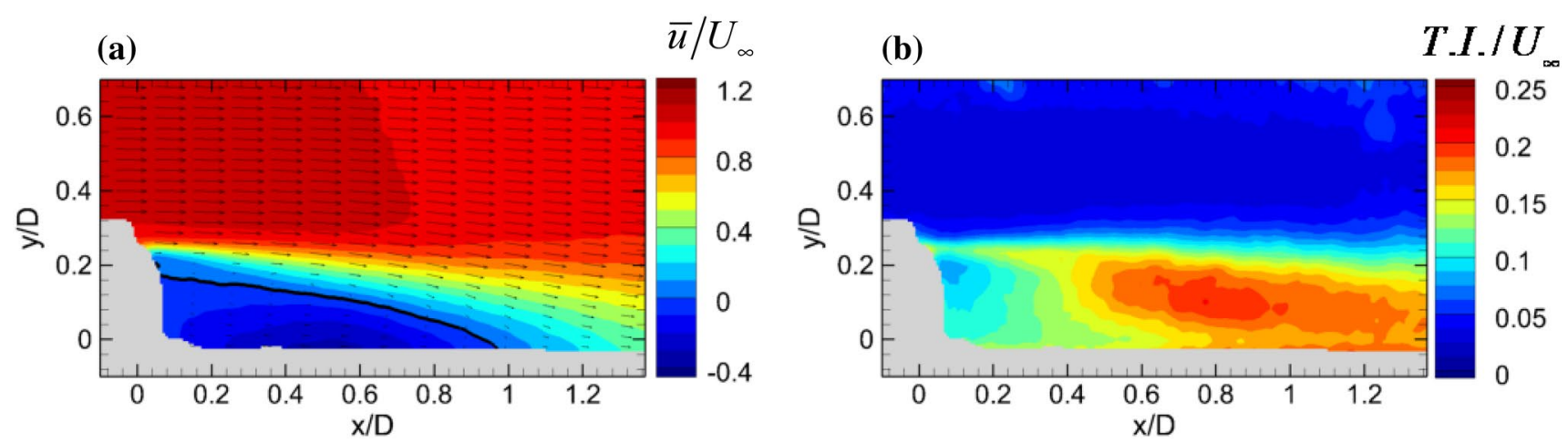

Fig. 7 Characterisation of the flow field statistics: a mean streamwise velocity and $\mathbf{b}$ turbulence intensity. Black line in figure a indicates zero streamwise velocity 
walls in the experiment. Rather, the difference in reattachment length is attributed to the lower freestream turbulence levels in the simulation. The lower turbulence levels lead to reduced mixing between low-momentum fluid in the recirculation region and high-momentum fluid in the freestream for a given streamwise position and, therefore, later reattachment. The presence of relatively high freestream turbulence levels in the experimental facility was demonstrated by measurements of pressure fluctuations in a test section without the main body of the model (not shown here for brevity).

\subsection{Pressure results}

The procedure to calculate the pressure fields from the PIV velocity data is similar as for the simulated experiment (Sect. 4.2), with the only difference that the pressure values applied as Dirichlet boundary condition at the top of the domain are determined from the relations for isentropic flow. The validity of using an isentropic flow model in the top of the domain was confirmed by van Gent et al. (2017a).

Figure 8 shows the pressure fields reconstructed using the different terms, as previously. Consistent with earlier observations, there is a relatively large difference between using mean flow terms only (top figure) and using both mean flow and Reynolds-stresses terms (middle figure). Additional consideration of the density-gradient terms (bottom figure) has a marginal impact (bottom figure). Quantitatively, the r.m.s. difference due to the addition of the Reynolds-stresses terms is $19 \%$ with respect to the r.m.s. of the mean flow term-only solution. Additional inclusion of density-gradient terms has an effect of only about $1 \%$.

To validate the reconstructed pressure results, Fig. 9 compares the PIV-based pressure distribution over the afterbody to transducer reference measurements. To obtain reference measurements at eight locations with four pressure transducers, the main body was translated with respect to the afterbody-support, such that the relative locations of the transducers shifted. PIV-based pressure profiles have been obtained at two vector spacing distance from the lower boundary of the measurement domain, since the data directly at the boundary were found to be noisy. The simulation data from the numerical assessment are included for comparison. The horizontal axis has been scaled by the reattachment length to account for the difference in reattachment length between the experiment and the simulation (1.0 vs. $1.2 \mathrm{D}$, respectively; see Sect. 5.2 for discussion).

The simulation data and the transducer measurements show excellent agreement. The PIV-based pressure profile calculated with Reynolds-stresses terms shows a good agreement with both the simulation data and the transducer measurements. The typical deviation is smaller than $\Delta C_{\mathrm{p}}<0.02$. Comparison of the different reconstructed profiles again shows the necessity of including the Reynolds-stresses terms
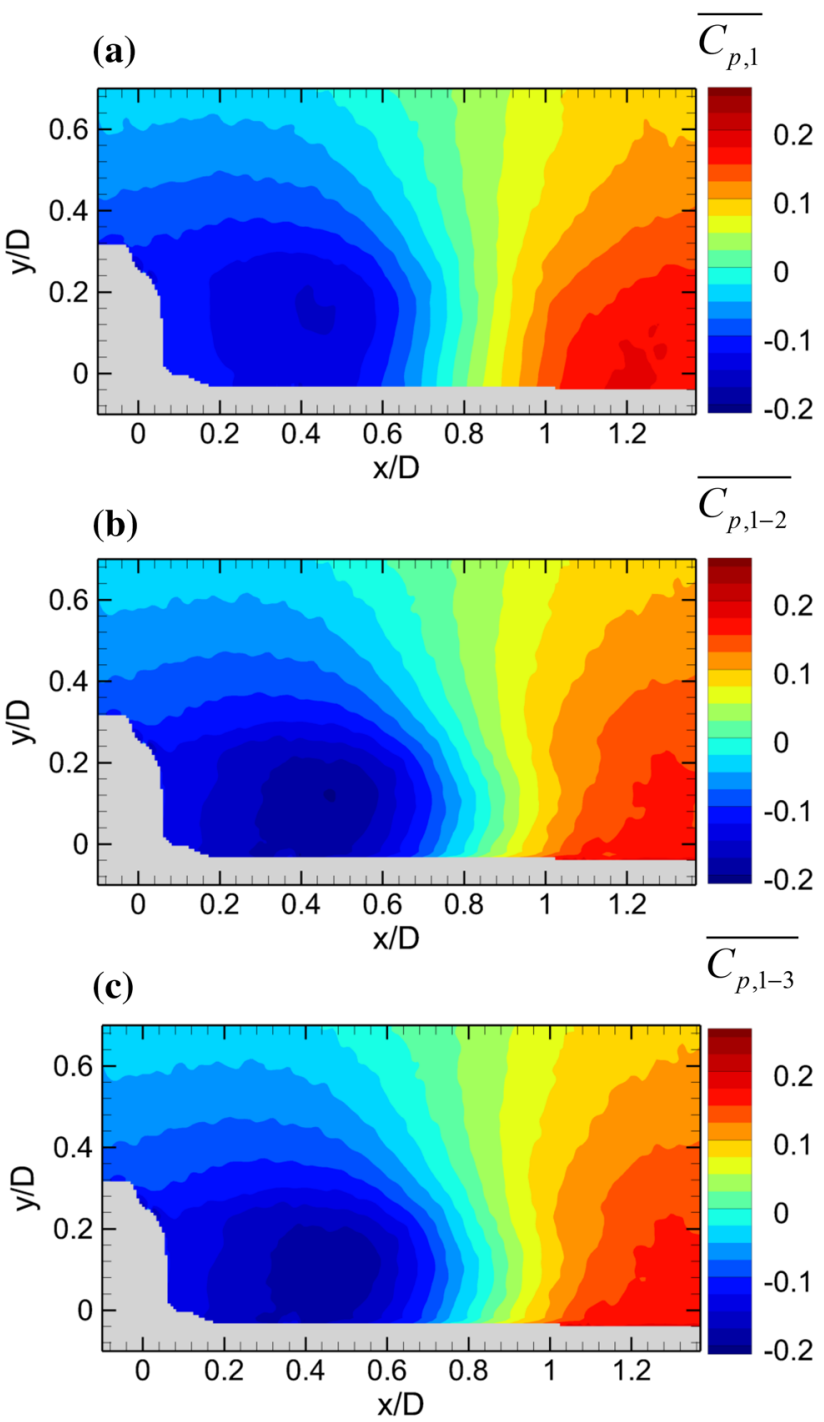

Fig. 8 Mean pressure field reconstructed a using mean flow terms, $\mathbf{b}$ using mean flow and Reynolds-stresses terms, and $\mathbf{c}$ using mean flow, Reynolds-stresses and density-gradient terms

and the negligible impact of accounting for density-gradient terms. Using the simulation data, it could be concluded that deviations between the PIV-based pressure profile and the other profiles can, to a large extent, be attributed to the distance from the afterbody at which the PIV-based pressure profile was obtained. This distance is the direct result of the challenge to obtain reliable PIV measurements close to the model due to laser light reflections. Although this was not a limiting factor here, in other experiments, the density of seeding particles near surfaces may be relatively low, posing an additional challenge.

The comparison of the transducer measurements and PIVbased pressure profile in Fig. 9 is sensitive to the value of the freestream velocity used in the PIV-based reconstruction. This is because the normalisation of the transducer 


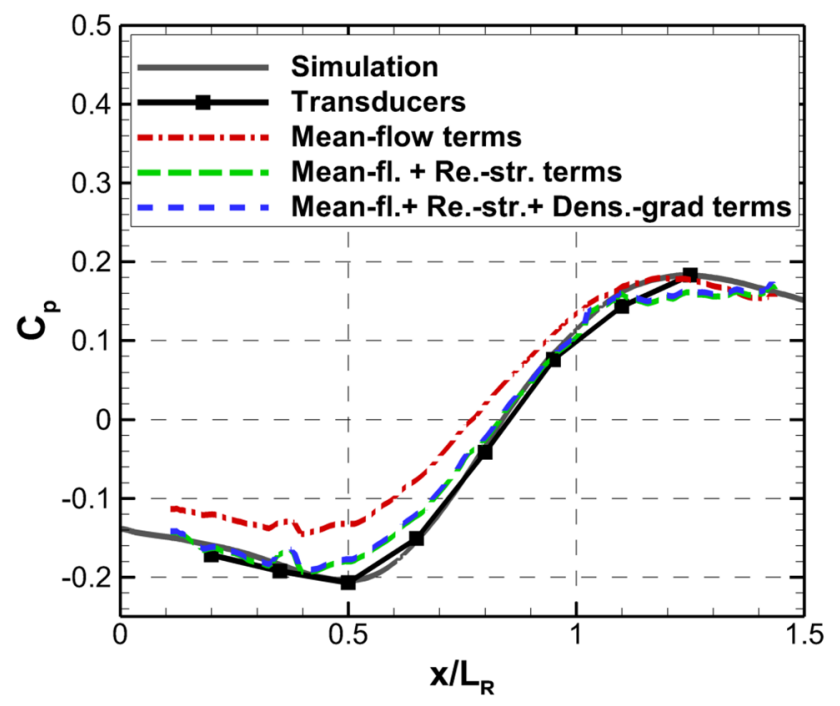

Fig. 9 Mean pressure distribution over the afterbody as obtained from PIV. The figure includes reference measurements from pressure transducers and simulation data (Sect. 3)

measurements only requires the freestream pressure $\left(p_{\infty}\right)$ and the freestream Mach number $\left(M_{\infty}\right)$, whereas the PIVbased pressure level is sensitive to the freestream Mach number $\left(M_{\infty}\right)$, pressure $\left(p_{\infty}\right)$, and velocity $\left(U_{\infty}\right)$. In the present study, the value of the static freestream pressure $\left(p_{\infty}\right)$ was obtained by static pressure measurements through a pressure tap in the wall of the test section. The freestream Mach number $\left(M_{\infty}\right)$ and the freestream velocity $\left(U_{\infty}\right)$ were then calculated via the isentropic flow relations, with the total temperature $\left(T_{\mathrm{t}}\right)$ and the total pressure $\left(p_{\mathrm{t}}\right)$ measured in the settling chamber as additional inputs. In short, the freestream parameters were calculated from direct measurements of the total temperature $\left(T_{\mathrm{t}}\right)$ and the total pressure $\left(p_{\mathrm{t}}\right)$ and the freestream pressure $\left(p_{\infty}\right)$. The above underlines the importance of good control and knowledge of the experimental conditions.

\section{Conclusions}

The computation of (mean) pressure fields from PIV data through the application of the momentum equation requires additional flow governing relations to be invoked in the case of compressible flow, to account for the variable density. A convenient solution consists of combining the gas law and the adiabatic flow assumption (as a specific form of the energy equation), together with the momentum equation with the viscous terms removed (van Oudheusden et al. 2007; Souverein et al. 2007; van Oudheusden 2008). In an earlier publication (van Oudheusden 2008), a Reynolds-averaging formulation for unsteady flow was developed, based on the assumption that density fluctuations can be neglected.
The present communication has addressed the accuracy of this assumption, by means of an evaluation of the relevance of the terms that are discarded. The assessment methodology involves a theoretical order-of-magnitude analysis, complemented with a quantitative assessment based on a simulated and a real PIV experiment of a transonic afterbody flow.

The assessments show that it is sufficient to account for spatial variations in the mean flow and the Reynolds-stresses and that temporal and spatial density variations (fluctuations and gradients) are of secondary importance and comparable order-of-magnitude. This has two interesting consequences in view of the practical implementation of the procedure of experimentally obtaining mean pressure from velocity statistics in compressible flows. First, the original formulation of the problem can be simplified to:

$\frac{\partial \ln \left(\bar{p} / p_{\infty}\right)}{\partial x_{i}}=-\frac{1}{R T}\left(\bar{u}_{j} \frac{\partial \overline{u_{i}}}{\partial x_{j}}+\frac{\overline{\partial u_{i}^{\prime} u_{j}^{\prime}}}{\partial x_{j}}\right)$.

Second, since the density-gradient term (which can be calculated but has been omitted) has been found to be of comparable order-of-magnitude as the terms related to the density fluctuations (which cannot be determined from the PIV data), comparing the results computed with and without density-gradient term can provide an indication of the error introduced by not accounting for the density fluctuations. This comparison permits the experimental procedure to validate the approximation of not accounting for density fluctuations without requiring to have access to "exact" reference pressure data.

Acknowledgements This work has been supported by the European FP7 project "NIOPLEX", Grant agreement 605151. The numerical simulation data on which the synthetic test case was based were provided by P.-É. Weiss of ONERA-Meudon. The PIV measurements used in the experimental test case were performed in collaboration with S.G. Brust.

Open Access This article is distributed under the terms of the Creative Commons Attribution 4.0 International License (http://creativeco mmons.org/licenses/by/4.0/), which permits unrestricted use, distribution, and reproduction in any medium, provided you give appropriate credit to the original author(s) and the source, provide a link to the Creative Commons license, and indicate if changes were made.

\section{References}

Atkinson C, Soria J (2009) An efficient simultaneous reconstruction technique for tomographic particle image velocimetry. Exp Fluids 47:553-568

Baur T, Köngeter J (1999) PIV with high temporal resolution for the determination of local pressure reductions from coherent turbulent phenomena. 3rd Int. workshop on PIV, Santa Barbara, CA

Blinde PL, Lynch KP, Schrijer FFJ, van Oudheusden BW (2015) Determination of instantaneous pressure in a transonic base flow 
using four-pulse tomographic PIV. 11th Int. Symp. Part. Image Velocim., PIV2015, Santa Barbara, CA

Charonko JJ, King CV, Smith BL, Vlachos PP (2010) Assessment of pressure field calculations from particle image velocimetry measurements Meas. Sci Technol 21:105401

de Kat R, van Oudheusden BW (2012) Instantaneous planar pressure determination from PIV in turbulent flow. Exp Fluids 52:1089-1106

Discetti S, Astarita T (2012) Fast 3D PIV with direct sparse crosscorrelations. Exp Fluids 53:1437-1451

Discetti S, Natale A, Astarita T (2013) Spatial filtering improved tomographic PIV. Exp Fluids 54:1505

Ghaemi S, Ragni D, Scarano F (2012) PIV-based pressure fluctuations in the turbulent boundary layer. Exp Fluids 53:1823-1840

Gurka R, Liberzon A, Hefetz D, Rubinstein D, Shavit U (1999) Computation of pressure distribution using PIV velocity data. 3rd Int. Workshop on PIV

Humble RA, Scarano F, van Oudheusden BW (2007) Unsteady flow organization of compressible planar base flows. Phys Fluids 19:076101

Jeon YJ, Chatellier L, Beaudoin A, David L (2015) Least-square reconstruction of instantaneous pressure field around a body based on a directly acquired material acceleration in time-resolved PIV. 11th Int. Symp. Part. Image Velocim., PIV2015, Santa Barbara, CA

Lynch KP, Scarano F (2014) Material acceleration estimation by fourpulse tomo-PIV. Meas Sci Technol 25:084005

Novara M, Schanz D, Reuther N, Kähler CJ, Schröder A (2016) Lagrangian 3D particle tracking in high-speed flows: shake-thebox for multi-pulse systems, Exp Fluids 57:128

Ragni D, Schrijer F, van Oudheusden BW, Scarano F (2010) Particle tracer response across shocks measured by PIV. Exp Fluids 50:53-64

Scarano F, Riethmuller ML (2000) Advances in iterative multigrid PIV image processing. Exp Fluids 29(7):S51-S60

Sciacchitano A, Wieneke W (2016) PIV uncertainty propagation. Meas Sci Technol 27:084006

Sibson R (1981) A brief description of natural neighbor interpolation (Chap. 2). In: Barnett V (ed) Interpreting multivariate data. Wiley, Chichester, pp 21-36

Smits AJ, Dussauge J-P (2006) Turbulent shear layers in supersonic flow, 2nd edn. Springer, New York

Souverein LJ, van Oudheusden BW (2009) Application of a dualplane particle image velocimetry (dual-PIV) technique for the unsteadiness characterization of a shock wave turbulent boundary layer interaction. Meas Sci Technol 20:074003

Souverein LJ, van Oudheusden BW, Scarano F (2007) Particle image velocimetry based loads determination in supersonic flows. AIAA Paper 2007-0050, 45th AIAA aerospace sciences meeting, Reno, NV

Unal MF, Lin JC, Rockwell D (1997) Force prediction by PIV imaging: a momentum based approach. J Fluids Struct 11:965-971

van Oudheusden BW (2008) Principles and application of velocimetrybased planar pressure imaging in compressible flows with shocks. Exp Fluids 45:657-674

van Oudheusden BW (2013) PIV-based pressure measurement. Meas Sci Technol 24:032001

van Oudheusden BW, Souverein LJ (2007) Evaluation of the pressure field from PIV in a shock wave boundary layer interaction, 7th international symposium on particle image velocimetry, Rome, Italy

van Oudheusden BW, Scarano F, Roosenboom EWM, Casimiri EWF, Souverein LJ (2007) Evaluation of integral forces and pressure fields from planar velocimetry data for incompressible and compressible flows. Exp Fluids 43:153-162

van Gent PL, Michaelis D, van Oudheusden BW, Weiss P-É, de Kat R, Laskari A, Jeon YJ, David L, Schanz D, Huhn F, Gesemann S, Novara M, McPhaden C, Rival D, Schneiders JFG, Schrijer FFJ (2017a) Comparative assessment of pressure field reconstructions from particle image velocimetry measurements and Lagrangian particle tracking. Exp Fluids 58:33

van Gent PL, Payanda Q, Brust SG, van Oudheusden BW, Schrijer FFJ (2017b) Experimental study to the effects of exhaust plume and nozzle length on transonic and supersonic axisymmetric base flows, EUCASS 2017, 7th European conference for aeronautics and space sciences, Milan, Italy

Violato D, Moore P, Scarano F (2011) Lagrangian and Eulerian pressure field evaluation of rod-airfoil flow from time-resolved tomographic PIV. Exp Fluids 50:1057-1070

Weiss P-É, Deck S, Robinet J-C, Sagaut P (2009) On the dynamics of axisymmetric turbulent separating/reattaching flows. Phys Fluids 21:075103

Westerweel J, Scarano F (2005) Universal outlier detection for PIV data. Exp Fluids 39:1096-1100

Wieneke B (2008) Volume self-calibration for 3D particle image velocimetry. Exp Fluids 45:549-556 